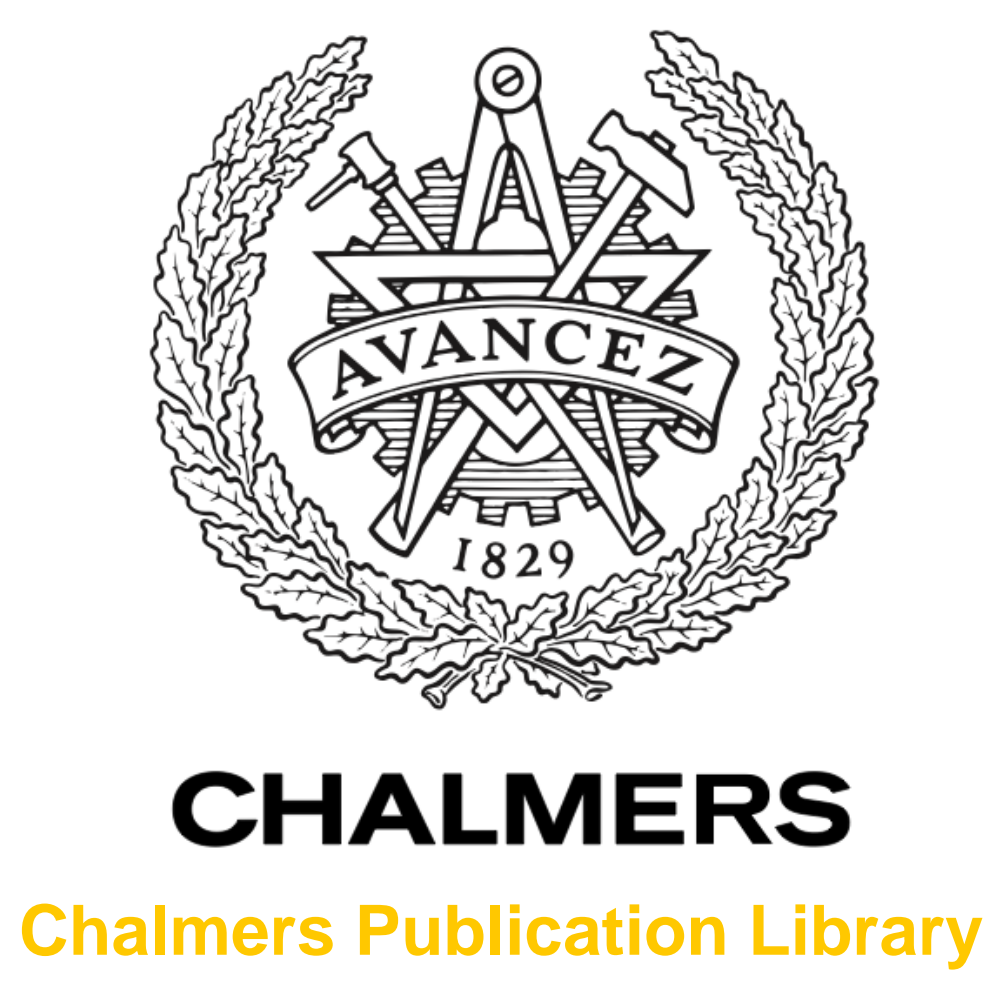

\title{
Resource allocation for V2X communications: A local search based 3D matching approach
}

This document has been downloaded from Chalmers Publication Library (CPL). It is the author's version of a work that was accepted for publication in:

\section{Proceedings IEEE International Conference on Communications}

Citation for the published paper:

Wei, Q. ; Wanlu, S. ; Bai, B. et al. (2017) "Resource allocation for V2X communications: A local search based 3D matching approach". Proceedings IEEE International Conference on

Communications

Downloaded from: http://publications.lib.chalmers.se/publication/250080

Notice: Changes introduced as a result of publishing processes such as copy-editing and formatting may not be reflected in this document. For a definitive version of this work, please refer to the published source. Please note that access to the published version might require a subscription. 


\title{
Resource Allocation for V2X Communications: A Local Search Based 3D Matching Approach
}

\author{
Qing Wei, Wanlu Sun, Student Member, IEEE, Bo Bai, Senior Member, IEEE, \\ Li Wang, Senior Member, IEEE, Erik G. Ström, Senior Member, IEEE, and Mei Song, Member, IEEE
}

\begin{abstract}
Vehicle-to-everything (V2X) communications, enabled by cellular device-to-device (D2D) links, have recently drawn much attention due to its potential to improve traffic safety, efficiency, and comfort. In this context, however, intracell interference combined with demanding latency and reliability requirements of safety vehicular users (V-UEs) are challenging issues. In this paper, we study a resource allocation problem among safety V-UEs, non-safety V-UEs, and conventional cellular UEs (C-UEs). Firstly, the resource allocation problem is formulated as a three-dimensional matching problem, where the objective is to maximize the total throughput of non-safety V-UEs on condition of satisfying the requirements on C-UEs and on safety V-UEs. Due to its NP-hardness, we then exploit hypergraph theory and propose a local search based approximation algorithm to solve it. Through simulation results, we show that the proposed algorithm outperforms the existing scheme in terms of both throughput performance and computational complexity.
\end{abstract}

Index Terms- $\mathrm{V} 2 \mathrm{X}$ communications, resource allocation, hypergraph, 3D matching, local search

\section{INTRODUCTION}

Recently, vehicle to everything (V2X) communications have drawn much attention due to their potential to improve traffic safety and efficiency. One important characteristic of this type of communications is its localized nature, i.e., requiring cooperation between vehicles and devices in close proximity. Besides, there are also strict latency and reliability requirements on safety related applications [1]- [3].

One popular legacy solution for $\mathrm{V} 2 \mathrm{X}$ communications is ad hoc communications over $802.11 \mathrm{p}$ standard. However, the lack of stringent quality of service (QoS) provisioning and absence of centralized management in the 802.11p standard make it challenging to satisfy safety application requirements. An alternative solution for V2X is to utilize direct device-todevice (D2D) links in the Long-term Evolution (LTE) cellular systems [4]- [6]. In this case, to further improve spectrum efficiency, underlay D2D network has been regarded as a promising enabler for V2X communications [7] [8].

Some researches have been dedicated to the V2X communications in the LTE cellular scenarios. In [9], a cooperative

Q. Wei, L. Wang (corresponding author), and M. Song are with the School of Electronic Engineering, Beijing University of Posts and Telecommunications, Beijing 100876, China. Dr. L. Wang is also with National Mobile Communications Research Laboratory, Southeast University, Nanjing, 210096, China email: liwang@bupt.edu.cn. B. Bai is with the Department of Electronics Engineering and Tsinghua National Laboratory for Information Science and Technology (TNList), Tsinghua University, Beijing 100084, China. W. Sun and E. G. Ström are with the Department of Signals and Systems, Chalmers University of Technology, Gothenburg, Sweden.

The work was supported in part by the NSFC of China (Grants No. 61571056 and No. 61372117), the open research fund of the National Mobile Communications Research Laboratory of Southeast University (Grant No. 2016D04), and the State Major Science and Technology Special Projects of China under Grant 2016ZX03001017-004. relaying mechanism is proposed to guarantee the channel quality of V2X communications. In works [4] and [10], the cloud-based servers are employed for the vehicular safety applications. In the V2X applications, the safety-related communications refer to the case that the messages transmitted require high QoS, such as congestion messages, emergency messages. Otherwise, the non-safety-related communications, such as short messages and entertainment video, are regarded as non-safety communications. In our scenario, we sort the V2X communications into safety V-UEs and non-safety VUEs according to these two types of communications. Thus the radio resource management (RRM) among the C-UEs, safety $\mathrm{V}$-UEs, and non-safety V-UEs is the focus of the paper.

In the context of $\mathrm{V} 2 \mathrm{X}$ underlaying systems, we assume that non-safety V-UEs can share the cellular resource blocks with the safety V-UEs simultaneously to transmit. Hence, one of the most critical challenges is the interference caused by resource reuse among the conventional cellular network, safety V-UEs, and non-safety V-UEs. In V2X communications, safety communications often require high reliability and low latency, and its message size is typically smaller than that in nonsafety communications [1]. Considering a more general case, the objective of our paper is to maximize the total rate of non-safety V-UEs. Hence, the key of the RRM strategy is to determine how the V-UEs reuse uplink resource blocks (RBs) with C-UEs. To tackle these challenges, a three-dimensional weighted matching problem is formulated to model the RRM problem among the C-UEs, safety V-UEs, and non-safety VUEs.

In this paper, we investigate the methods for multidimensional matching problems. Most existing methods fall into two categories: 1) transform the multidimensional matching problem into the two-dimensional matching problems, i.e., the IHM in [11], the pairing algorithm in [12], and the parallel implementation of Hopcroft-Karp in [13]; 2) construct the hypergraph model [14]- [17] or $k$-set packing problem [19][22]. Among the methods applied in the latter, local search has been used successfully to solve such computationally hard optimization problem and studied in many research domains [23]- [25].

The local search is proposed as an effective technique for designing high performance approximation algorithm for computationally hard problems. Generally, we start with a feasible solution and then try to improve the solution in moving to a neighboring solution under some optimization criterion. Given a ratio for the optimal objective value and the current achievable one, if it is always lower than $\rho$ for every instance of the problem, the $\rho$ can be defined as the approximation ratio [19]. For $k$-dimensional matching, a simple greedy algorithm returning a set of matching among different UEs gives a $k$ approximation ratio [21], and a local search algorithm can obtain better approximation [20]- [22]. The best known polynomial 
time $^{1}$ approximation ratio is $(k+\varepsilon) / 2$ in [20]. There, $\varepsilon>0$ and $k$ is an integer greater than or equal to 3 .

The hypergraph matching theory [26] is employed to formulate the three-dimensional matching problem. A hypergraph is a set of vertices and a family of subsets of vertices, each referred as a hyperedge. In our scenario, the C-UEs, safety V-UEs, and non-safety V-UEs are partite, and the primary $k$ dimensional matching problem can also be regarded as $k$-set packing problem. Specially, given a family of sets, the problem to find a maximal sub-family of sets that are pairwise disjoint in the problem of $k$-set packing is equivalent to the problem of finding a maximal matching in a hypergraph [27]. Hence, a local search based three-dimensional matching approach is desired to be established from the hypergraph model.

The novelty of our work mainly lies in introducing a 3D-matching based V2X resource allocation algorithm (3DVRAA) to solve the RRM problem joint with safety V-UEs, non-safety V-UEs, and C-UEs. The main contributions of this paper are summarized as follows:

- formulation of a joint RRM problem for safety V-UEs, non-safety V-UEs, and conventional C-UEs;

- construction of a hypergraph model for the associated RRM problem;

- proposed a 3D-matching based algorithm to solve the RRM problem;

- performance evaluation of the proposed scheme with other methods.

In the remainder of the paper, Section II presents the system model and formulates the RRM problem as a 3D matching problem. In Section III, we decompose the primary problem, construct its associated hypergraph model, and propose a 3Dmatching based V2X resource allocation algorithm to solve it. In Section IV, simulation results are presented and discussed. Finally, Section V concludes the paper.

\section{System Model and Problem Formulation}

\section{A. System Model for V2X Communications}

As shown in Fig. 1, we consider a single cell V2X communications scenario with $M$ C-UEs, $K$ safety V-UEs and $Q$ non-safety V-UEs. The V-UEs communicate by reusing the uplink resource of C-UEs, and thus interference exists among different types of UEs.

For conventional C-UEs, which is denoted by $\mathcal{M}=$ $\{1,2, \ldots, M\}$, orthogonal $\mathrm{RB}$ allocation is applied in the uplink. Meanwhile, the set of safety V-UEs, and non-safety VUEs are denoted by $\mathcal{K}=\{1,2, \ldots, K\}$ and $\mathcal{Q}=\{1,2, \ldots, Q\}$, respectively. Orthogonal Frequency Division Multiple Access (OFDMA) is employed to support multiple access for both the cellular and V2X communications, where a set $\mathcal{F}$ of RBs are available for resource allocation, and $\mathcal{F}=\{1,2, \ldots, F\}$. In this system, the base station (BS) coordinates the resource allocation among C-UEs, safety V-UEs and non-safety V-UEs. The channel gains among C-UEs, safety V-UEs, and non-safety V-UEs are summarized in TABLE 1 . Note that the $g_{k, q}^{K, Q}$ and $g_{q, k}^{Q, K}$ are equal in amount due to channel reciprocity.

To perform RRM, the BS needs certain level of channel state information (CSI) for all these involved links. Note that the channel power gains are measured at a long-term level such that the fast fading efficient has been averaged out. Also, the thermal noise satisfies independent Gaussian distribution with zero mean and variance $\sigma^{2}$.

${ }^{1}$ An algorithm is called polynomial time algorithm if its running time is polynomial in the size value of the input.

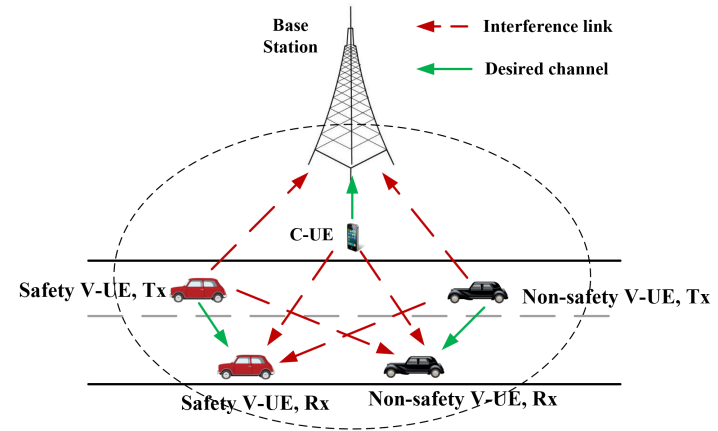

Fig. 1. System Model.

TABLE I

Summary of Channel Average Power Gains

\begin{tabular}{ll}
\hline Symbol & Definition \\
\hline$h_{m}^{M}$ & Desired channel average power gain of C-UE $m$, \\
$h_{k}^{K}$ & Desired channel average power gain of safety V-UE $k$, \\
$h_{q}^{Q}$ & Desired channel average power gain of non-safety V-UE $q$, \\
$g_{k}^{K}$ & Interference channel average power gain from safety V-UE $k$ to BS, \\
$g_{q}^{Q}$ & Interference channel average power gain from non-safety V-UE $q$ to BS, \\
$g_{m, K}^{M, k}$ & Interference channel average power gain from C-UE $m$ to safety V-UE $k$, \\
$g_{m, Q}^{M,}$, & Interference channel average power gain from C-UE $m$ to safety V-UE $q$, \\
$g_{k, Q}^{K, Q}$ & Interference channel average power gain from safety V-UE $k$ to non-safety V-UE $q$, \\
$g_{q, k}^{Q, K}$ & Interference channel average power gain from safety V-UE $q$ to non-safety V-UE $k$. \\
\hline
\end{tabular}

\section{B. Problem Formulation}

We assume that the RB of the C-UE can be shared by at most one safety V-UE and one non-safety V-UE simultaneously, and define a binary variable $x_{m, f}^{M}$ for allocation of C-UEs as

$$
x_{m, f}^{M}= \begin{cases}1, & \text { when C-UE } m \text { is allocated to RB } f, \\ 0, & \text { otherwise. }\end{cases}
$$

Also, the allocation variables of safety V-UEs and non-safety V-UEs are denoted by $x_{k, f}^{K}$ and $x_{q, f}^{Q}$, which are defined as

$x_{k, f}^{K}= \begin{cases}1, & \text { when safety V-UE } k \text { is allocated to RB } f, \\ 0, & \text { otherwise. }\end{cases}$

and

$x_{q, f}^{Q}=\left\{\begin{array}{l}1, \quad \text { when non-safety V-UE } q \text { is allocated to RB } f, \\ 0,\end{array}\right.$ $x_{q, f}= \begin{cases}0, & \text { otherwise. }\end{cases}$

respectively.

We assume that each RB can not be shared by two or more UEs in the same type. Thus the orthogonality among the same type of UEs can be described as

$$
\begin{aligned}
& \sum_{q=1}^{Q} x_{q, f}^{Q} \leq 1, \sum_{k=1}^{K} x_{k, f}^{K} \leq 1, \forall f \in \mathcal{F}, \\
& \sum_{m=1}^{M} x_{m, f}^{M} \leq 1, \sum_{f=1}^{F} x_{m, f}^{M}=E_{m}^{M}, \forall m \in \mathcal{M} .
\end{aligned}
$$

where the $E_{m}^{M}$ stands for the number of RBs assigned to C-UE $m$, and it satisfies the minimum required number of RBs for C-UEs.

Furthermore, we define $\gamma_{q, f}^{Q}, \gamma_{k, f}^{K}$ and $\gamma_{m, f}^{M}$ as the signalto-interference-and-noise ratios (SINRs) of non-safety V-UE $q$, safety V-UE $k$ and C-UE $m$ on the RB $f$, which can be calculated as 


$$
\begin{gathered}
\gamma_{q, f}^{Q}=\frac{p_{q, f}^{Q} h_{q}^{Q}}{\sigma^{2}+p_{k, f}^{K} g_{k, q}^{K, Q}+p_{m, f}^{M} g_{m, q}^{M, Q}}, \forall f \in \mathcal{F}, q \in \mathcal{Q} \\
\gamma_{k, f}^{K}=\frac{p_{k, f}^{K} h_{k}^{K}}{\sigma^{2}+p_{m, f}^{M} g_{m, k}^{M, K}+p_{q, f}^{Q} g_{q, k}^{Q, K}}, \forall f \in \mathcal{F}, k \in \mathcal{K} \\
\gamma_{m, f}^{M}=\frac{p_{m, f}^{M} h_{m}^{M}}{\sigma^{2}+p_{k, f}^{K} g_{k}^{K}+p_{q, f}^{Q} g_{q}^{Q}}, \forall f \in \mathcal{F}, m \in \mathcal{M}
\end{gathered}
$$

The optimization variables $p_{q, f}^{Q}\left(p_{k, f}^{K}\right.$ and $\left.p_{m, f}^{M}\right)$ represents the transmission power of non-safety V-UE $q$ (safety V-UE $k$ and C-UE $m$ ) on the RB $f$. We consider the case where the C-UEs and safety V-UEs should be guaranteed a certain QoS, while the non-safety V-UEs will receive a best effort service. To be precise, we assume that the QoS requirement for the C-UE $m$ will be satisfied if $\gamma_{m, f}^{M} \geq \gamma^{M}$ for all RBs allocated to the $m$-th C-UE (i.e., for the RB $f$ such that $x_{m, f}^{M}=1$ ). Similarly, we assume that the QoS for the safety V-UE $k$ is satisfied if $\gamma_{k, f}^{K} \geq \gamma^{K}$ for the RB allocated to the $k$-th safety VUE (i.e., for the RB $f$ such that $x_{k, f}^{K}=1$ ). We then proceed to maximize the sum rate of the non-safety V-UEs. The resulting optimization problem $\mathcal{P}_{1}$ can be written as (3).

$$
\begin{aligned}
\mathcal{P}_{1}: \max _{p_{q, f}^{Q}, p_{k, f}^{K}, p_{m, f}^{M},} & \sum_{q=1}^{Q} \sum_{f=1}^{F} \log _{2}\left(1+\gamma_{q, f}^{Q}\right) \\
\text { s.t. } \quad & \text { Constraints (1a) and (1b), } \\
& 0 \leq p_{q, f}^{Q} \leq x_{q, f}^{Q} P_{\max }^{Q}, \forall q \in \mathcal{Q}, f \in \mathcal{F}, \\
& 0 \leq p_{k, f}^{K} \leq x_{k, f}^{K} P_{\max }^{K}, \forall k \in \mathcal{K}, f \in \mathcal{F}, \\
& 0 \leq p_{m, f}^{M} \leq x_{m, f}^{M} P_{\max }^{M}, \sum_{f=1}^{F} p_{m, f}^{M} \leq P_{\max }, \\
& \gamma_{k, f}^{K} \geq \gamma^{K}, \gamma_{m, f}^{M} \geq \gamma^{M}, \forall f \in \mathcal{F} .
\end{aligned}
$$

where the $\gamma^{K}$ and $\gamma^{M}$ are the SINR thresholds for safety V-UEs and C-UEs. The individual maximum transmit power on each used RB for non-safety V-UEs, safety V-UEs, and C-UEs are $P_{\max }^{Q}, P_{\max }^{K}$ and $P_{\max }^{M}$, and $P_{\max }$ is the sum power constraint for C-UEs. Constraint (3c) (constraint (3d) and constraint (3e)) forces the transmit power of the non-safety V-UE $q$ (the safety V-UE $k$ and the C-UE $m$ ) on the RB $f$ to be 0 in case $x_{q, f}^{Q}=0\left(x_{k, f}^{K}=0\right.$ and $\left.x_{m, f}^{M}=0\right)$.

The optimization problem (3) is a maximum weighted matching (MWM) problem with nonlinear constraints, which is NP-hard [13]. When there exist a large number of VUEs, exhaustive search becomes unacceptable due to its high computational complexity. To our knowledge, the local search idea has been successfully used in designing approximate and efficient method for such problem. Therefore, we firstly decompose the primary problem and optimize the data rate of non-safety V-UEs on each RBs. Then, a local search based scheme is proposed to address the RRM problem.

\section{Problem Decomposition And Algorithm Design}

In the section, the optimization problem is decomposed into three stages. In Stage 1, we relax the constraints in (3) and consider a sub-optimal problem, in which we obtain the data rate of each non-safety V-UEs on different RBs. In Stage 2,

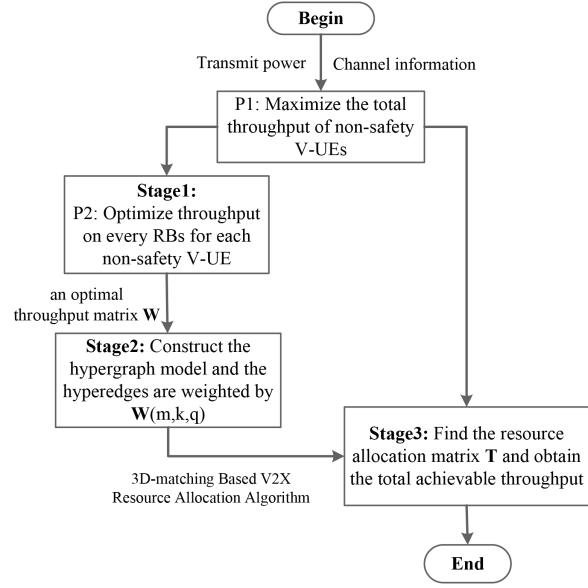

Fig. 2. Three Stages of Resource Allocation

we construct a hypergraph model for the RRM problem, and the hyperedges are weighted by the data rate obtained from Stage 1. We finally solve the RRM problem with the proposed algorithm and obtain the total achievable throughput of all the non-safety V-UEs in Stage 3. The procedure is shown in Fig. 2.

\section{A. Sub-problem for Optimal Resource}

In Stage 1, we relax the transmit power constraints in (3e) for all C-UEs into the individual power constraint, and it can be described as

$$
0 \leq p_{m, f}^{M} \leq x_{m, f}^{M} P_{\max }^{M} .
$$

Under the fairness consideration of SINRs, we optimize the data rate of each non-safety V-UE on every RB. Namely, the binary variables $x_{q, f}^{Q}, x_{k, f}^{K}$ and $x_{m, f}^{M}$ are 1 simultaneously, which means that the C-UE $m$, safety V-UE $k$ and non-safety $\mathrm{V}$-UE $q$ are assigned to the RB $f$. The sub-optimal problem $\mathcal{P}_{2}$ is formulated as (5).

$$
\begin{aligned}
\mathcal{P}_{2}: \max _{p_{q, f}^{Q}, p_{k, f}^{K}, p_{m, f}^{M}} & \log _{2}\left(1+\frac{p_{q, f}^{Q} h_{q}^{Q}}{\sigma^{2}+p_{k, f}^{K} g_{k, q}^{K, Q}+p_{m, f}^{M} g_{m, q}^{M, Q}}\right) \\
\text { s.t. } \quad & 0 \leq p_{q, f}^{Q} \leq P_{\max }^{Q}, \\
& 0 \leq p_{k, f}^{K} \leq P_{\max }^{K}, \\
& 0 \leq p_{m, f}^{M} \leq P_{\max }^{M}, \\
& \frac{p_{k, f}^{K} h_{k}^{K}}{\sigma^{2}+p_{m, f}^{M} g_{m, k}^{M, K}+p_{q, f}^{Q} g_{q, k}^{Q, K}} \geq \gamma^{K} \\
& \frac{p_{m, f}^{M} h_{m}^{M}}{\sigma^{2}+p_{k, f}^{K} g_{k}^{K}+p_{q, f}^{Q} g_{q}^{Q}} \geq \gamma^{M}
\end{aligned}
$$

where the $p_{q, f}^{Q}, p_{k, f}^{K}$, and $p_{m, f}^{M}$ are the optimization variables. Since the objective function in (5) is not concave with respect to $p_{k, f}^{K}$ and $p_{m, f}^{M}$, the problem is not convex. Nevertheless, the objective function is monotonically non-increasing in terms of $p_{k, f}^{K}$ and $p_{m, f}^{M}$, and thus we can eliminate them by achieving the equalities of the constraints (5e) and (5f). Then, the remaining problem is transformed into an equivalent convex optimization problem. The method in [7] can be applied to obtain the data 
rate of every non-safety V-UE in all possible combination of C-UEs, safety V-UEs, and non-safety V-UEs on RBs.

To make it concise, we define $W_{M \times K \times Q} \in R^{M \times K \times Q}$ as an $M \times K \times Q$ optimal throughput matrix. $W(m, k, q)$ denotes the data rate of non-safety V-UE $q$, which shares the RB with the C-UE $m$ and safety V-UE $k$, for $m \in \mathcal{M}, k \in \mathcal{K}$ and $q \in \mathcal{Q}$.

\section{B. Hypergraph Model and Conflict Graph}

In Stage 2, we define $T_{M \times K \times Q} \in R^{M \times K \times Q}$ as an $M \times K \times Q$ allocation matrix for general case. $T_{k, q}^{m}$ denotes the allocation binary variable for $m \in \mathcal{M}, k \in \mathcal{K}$ and $q \in \mathcal{Q}$, which is described as

$$
T_{k, q}^{m}= \begin{cases}1, & x_{m, f}^{M}=x_{k, f}^{K}=x_{q, f}^{Q}=1 \\ 0, & \text { otherwise. }\end{cases}
$$

Note that the constraints in (1) assume that each RB can not be shared with two or more UEs in the same type, thus we can obtain that

$$
\begin{aligned}
& \sum_{k \in \mathcal{K}} \sum_{q \in \mathcal{Q}} T_{k, q}^{m} \leq 1, \forall m \in \mathcal{M}, \\
& \sum_{m \in \mathcal{M}} T_{k, q}^{m} \leq 1, \forall k \in \mathcal{K}, q \in \mathcal{Q} .
\end{aligned}
$$

Thus, to maximize the total throughput of non-safety V-UEs, (3) is transformed into the problem to find an allocation matrix $T$ on throughput matrix $W$, which is a three-dimensional weighted matching problem. In the following, the concept of hypergraph will be firstly introduced.

A hypergraph $H$ is a pair $H=(V, E)$, where $V$ is the set of vertices and $E$ is the set of hyperedges [26]. In our discussed scenario, we denote the set of C-UEs, safety V-UEs and nonsafety V-UEs as $\mathcal{M}, \mathcal{K}$ and $\mathcal{Q}$ respectively, where $\mathcal{M}, \mathcal{K}, \mathcal{Q} \subseteq$ $V$. The combination $(m, k, q)$, where $m \in \mathcal{M}, k \in \mathcal{K}$ and $q \in \mathcal{Q}$, stands for a hyperedge $e \in E$ that intersects exactly one vertex from every vertex class. The hyperedge $(m, k, q)$ are weighted by $W(m, k, q)$, which is obtained from Stage 1 . The hypergraph matching aims at finding a subset $\mathcal{A} \subseteq E$ with the largest sum weights of hyperedges in $E$.

In Fig. 3(a), for example, we present all the hyperedges of the original hypergraph, and the Fig. 3(b) shows the feasible hyperedges under the constraints of (5b)-(5f), in which we use four closed curves to present the hyperedges. Given a hyperedge $e_{1} \in E$, if there exist other hyperedges that intersect with it at least one vertex, they will be regarded as the neighbours of $e_{1}$, denoted as $N\left(e_{1}\right)$. In Fig. 4(a), the $N\left(e_{1}\right)=N(1,1,2)=\{(3,3,2)\}$.

The conflict graph [19] of hypergraph $H$ is the graph where every hyperedge $e$ is denoted by a vertex $u \in U$, and the weight of each vertex corresponds to the weight of the related hyperedge. In Fig. 4(a), we use $u_{1}$ to denote the hyperedge $(1,1,2)$, which represents the combination of the 1-th C-UE, 1-th safety V-UE and 2-nd non-safety V-UE. If the hyperedges which are denoted as vertices are neighbours in $E$, these corresponding vertices are adjacent. After connecting all the adjacent vertices, we can obtain the conflict graph of the original hypergraph. For convenience, we divide all the vertices $U$ into two parts, $U_{A}$ and $U_{B}$. It assumes that the set $U_{A}$ is the initial independent set, and the set $U_{B}$ contains all the adjacent vertices of $U_{A}$.

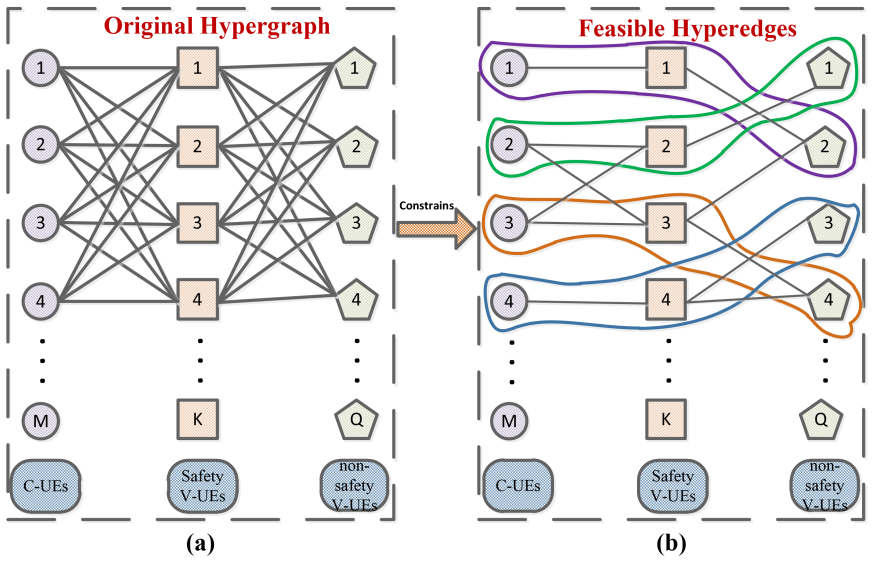

Fig. 3. Original Hypergraph and Feasible Hyperedges.

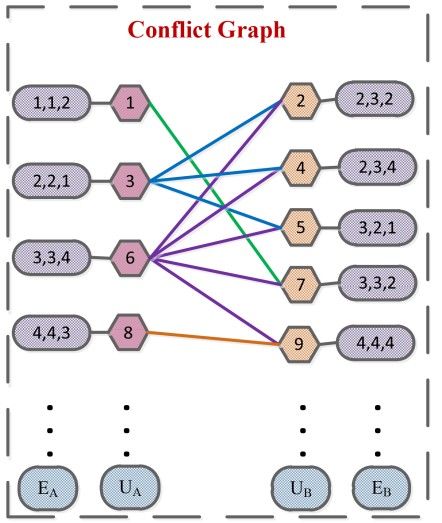

(a)

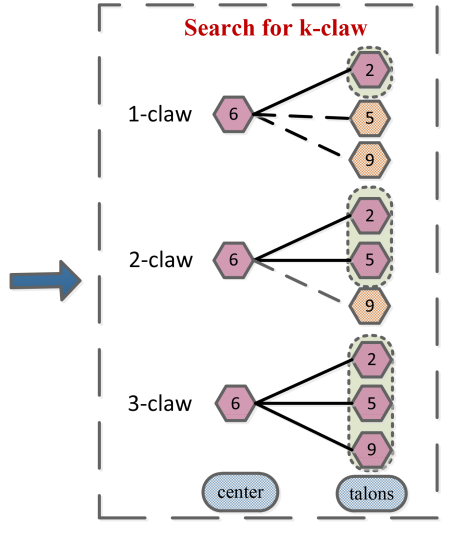

(b)
Fig. 4. Conflict Graph and Search for $k$-claw.

\section{Algorithm Design Based on k-claw}

The proposed local search approximate strategy is designed based on the [22], which can obtain a solution with approximation ratio $(k+1+\varepsilon) / 2$ in polynomial time for a $k$ dimensional matching problem. In this section, we will make a detailed introduction for the 3D-matching based V2X resource allocation algorithm used in Stage 3.

Definition. ( $k$-claw $C$ ) The $k$-claw is an induced subgraph $C$ that consists of an independent set $T_{c}$ of $k$ nodes, called talons, and a center node that is connected to all the talons. Specially, a graph is $k$-claw free if it possesses no $k$-claws.

Under the assumption that each hyperedge intersects $e_{1}$ in a distinct element, there can be at most $k$ hyperedges in $N\left(e_{1}\right)$ that are mutually disjoint, which means that there can be at most $k$ adjacent vertices for a fixed vertex in the conflict graph. In Fig. 4(a), $k=3, u_{3}$ has three adjacent vertices and $u_{6}$ can find 5 adjacent vertices. However, the $u_{3}$ can only find 1-claw and 2-claw, in case that $u_{2}$ and $u_{4}$ occupy the same elements. For comparison, the $u_{6}$ can find the 3-claw further. In the Figure. 4(b), we regard the $u_{6}$ as the center of a claw, and show the 1-claw, 2-claw, and 3-claw in order. It should be noted that we choose the $u_{2}$ as the 1-claw for instance, and the $u_{4}$ can also be regarded as the 1-claw at the beginning. Our algorithm structure based on the $k$-claw is designed and described in Algorithm 1. 


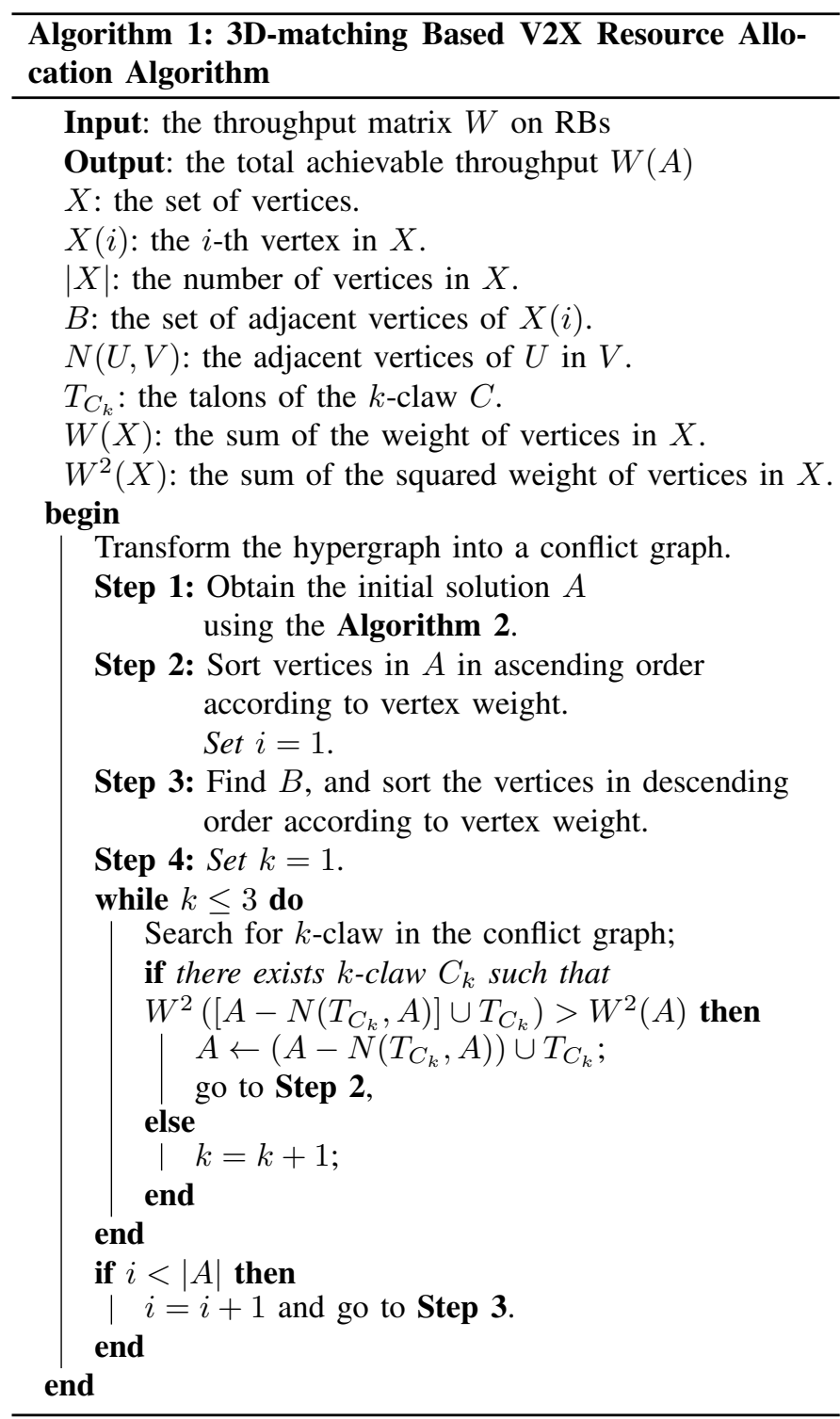

\section{Numerical Results And Performance Analysis}

We assume a single cell outdoor system with a carrier frequency of $800 \mathrm{MHz}$. There are $40 \mathrm{RBs}$, of which the bandwidth is $180 \mathrm{kHz}$ for uplink communications. In particular, we consider test case 2 defined by METIS [28], which describes an urban environmental model. In this topology, the entire region is a $500 \mathrm{~m} \times 500 \mathrm{~m}$ square and the size of each building is 120 $\mathrm{m} \times 120 \mathrm{~m}$. The antenna height is $26 \mathrm{~m}$ at the eNB and 1.5 $\mathrm{m}$ at each UE. The intended broadcast range of each vehicle is $18 \mathrm{~m}$.

The used channel models are also specified by [28], which describes the large scale modeling for different propagations scenarios (PSs). Specifically, we refer to PS $\sharp 3$ for the links connected to the eNB (i.e., $h_{m}^{M}, g_{k}^{K}$, and $g_{q}^{Q}$ ). The links between UEs (i.e., $h_{k}^{K}, h_{q}^{Q}, g_{m, q}^{M, Q}$, and $g_{k, q}^{K, Q}$ ) are based on $\mathrm{PS} \sharp 9$ in [28]. The maximum transmit power $P_{\max }^{M}$ and $P_{\max }^{K}$ are $24 \mathrm{dBm}$, and $P_{\max }^{Q}$ is $20 \mathrm{dBm}$. The white noise power is $-117 \mathrm{dBm}$.
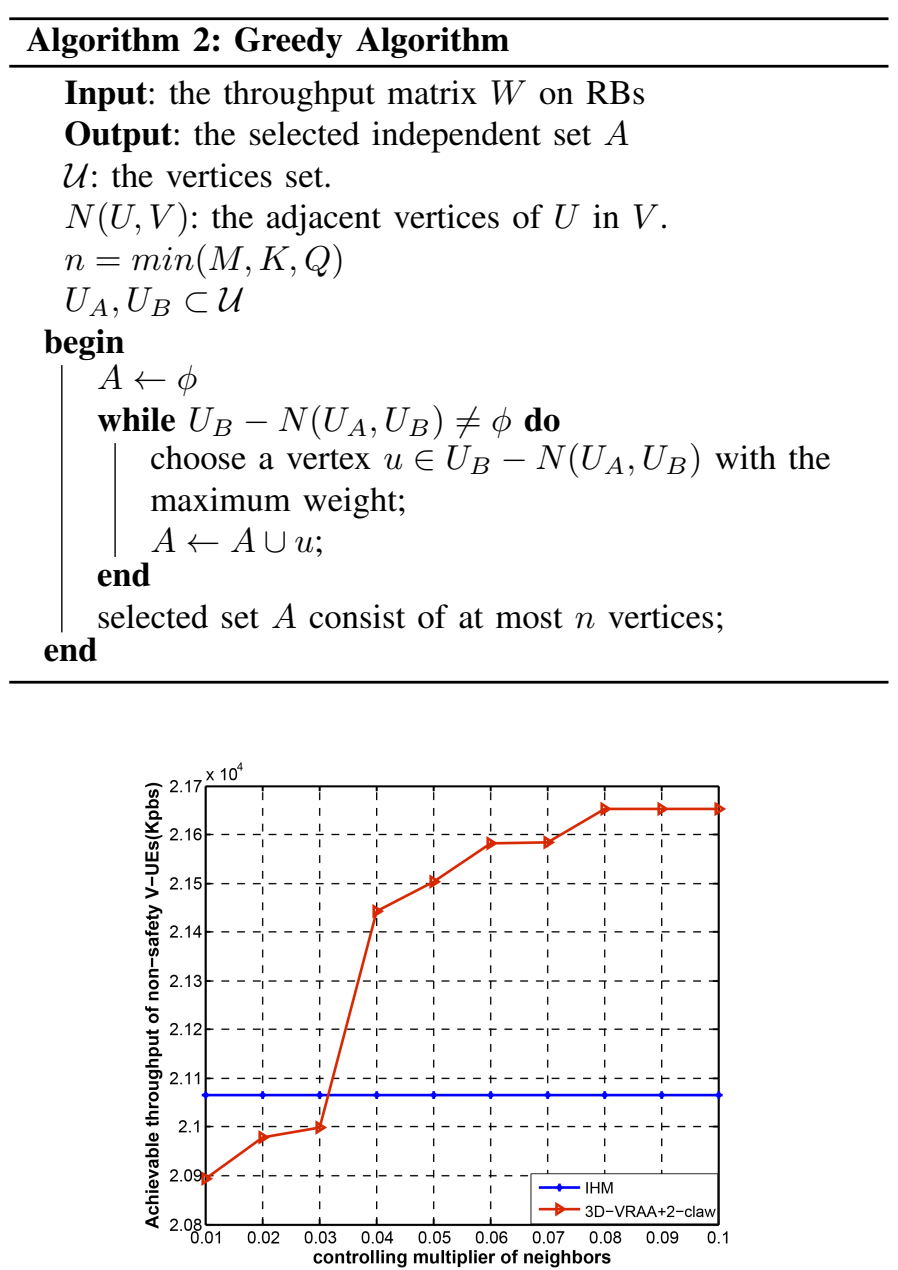

Fig. 5. Achievable throughput vs. controlling multiplier of neighbours.

To evaluate the performance improvement for adding $k$-claw to the algorithm, we search for the 1-claw, 2-claw, and 3claw in order. It shows that there is no much improvement in performance considering the adding of 3-claw, the comparison result of which is omitted due to the space limitation. Thus, the proposed algorithm terminates with the 2-claw search, and the following analysis of the 3D-matching based V2X resource allocation algorithm are all based on the 2-claw. Particularly, we compare our proposed algorithm with the IHM [11].

Since the set of adjacent vertices in $B$ has been sorted in a descending order, the search range $L^{\prime}$ of the $B$ will have an impact on the achievable performance and complexity of the algorithm. We define $L^{\prime}$ as $L^{\prime}=t \times L$, where $L$ is the total number of adjacent vertices in $B$, and $t$ is the controlling multiplier ranging from 0 to 1 . Fig. 5 illustrates that with a larger $t$, the performance of the IHM keeps unchanged, but the throughput of the proposed algorithm increases. It can be seen that when $t$ is greater than 0.04 , the performance of our algorithm is better than IHM. Furthermore, the achievable throughput converges into a stable level as $t$ increases.

Specially, the IHM algorithm transforms the threedimensional matching problem into an iterative twodimensional matching problem. Given $j$ iterations, the computation complexity of the IHM is $O\left(j \times S^{3}\right)$, and $S=$ $\max (M, K, Q)$ [11]. Note that in the given scenario, the $\max (M, K, Q)$ is 40 . Meanwhile, given $n$ iterations, the com- 


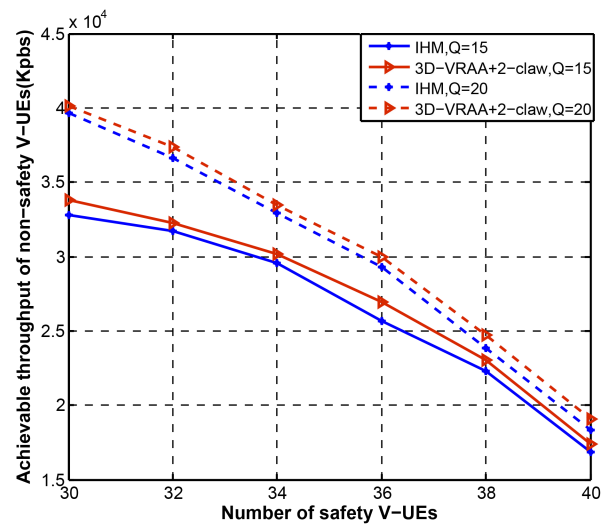

Fig. 6. Throughput comparisons of different schemes vs. number of safety V-UEs and non-safety V-UEs.

putation complexity of the proposed algorithm is $O\left(n \times L^{\prime 2}\right)$. Considering the maximum number of vertices in $B$, the $L$ can be calculated as 4680 . We set $t=0.05$ to guarantee the performance of the proposed algorithm based on Fig. 5, thus the $L^{\prime}$ is 234. If $n$ is not larger than $j$, the computation complexity of the proposed algorithm will show an advantage over the IHM. In addition, a V2X based distributed storage system enables computing offloading for challenging computation tasks, and the computation for the proposed algorithm can be executed in the data control center with greater computing ability. Therefore, the corresponding computation time is inclined to be modest.

Fig. 6 demonstrates the performance of different algorithms with different number of safety V-UEs and non-safety V-UEs. It can be seen that, due to the interference among the VUEs, the achievable throughput of non-safety V-UEs descends approximately linearly with the increasing number of safety V-UEs. With a fixed number of safety V-UEs, the increase of non-safety V-UEs leads to a higher achievable throughput. Notice that the performance of our proposed algorithm is better than IHM.

\section{CONCLUSION}

The paper investigates the resource allocation problem for D2D enabled V2X communications, where RBs are shared among safety V-UEs, non-safety V-UEs, and conventional CUEs. The resource allocation problem is formulated as a threedimensional matching problem that is known to be NP-hard. Hence, to solve it, we decompose the original problem into three stages and propose a local search based approximation solution. As illustrated by simulation results and analysis, the proposed algorithm shows improved throughput performance with reduced complexity. In the future, we will continue the design of $L^{\prime}$ such that the performance is maintained with further reduced computational complexity.

\section{REFERENCES}

[1] "3GPP TR 22.885 v1.0.0, Study on LTE support for V2X Services (Release 14)," Tech. Rep., Sep. 2015. [Online]. Avalible:http://www.3gpp.org/

[2] J. B. Kenney, "Dedicated short-range communications (DSRC) standards in the United States," In Proceedings of the IEEE, vol. 99, no. 7, pp. 1162-1182, Jul. 2011.

[3] Q. Yang and H. Wang, "Toward trustworthy vehicular social networks," IEEE Communication Magazine, vol. 53, no. 8, pp. 42-47, Aug. 2015.
[4] S. Kato, M. Hiltunen, K. Joshi, and R. Schlichting, "Enabling vehicular safety applications over LTE networks," in Proc. 2013 International Conference on Connected Vehicles and Expo, Las Vegas, NV, USA, Dec. 2013, pp. 747-752.

[5] L. Wang and G. Stuber, "Pairing for resource sharing in cellular deviceto-device underlays," IEEE Network, vol. 30, no. 2, pp. 122-128, Mar. 2016.

[6] L. Wang, H. Tang, and M. Cierny, "Device-to-device link admission policy based on social interaction information," IEEE Trans. Veh. Technol., vol. 64, no. 9, pp. 4180-4186, Sep. 2015.

[7] W. Sun, D. Yuan, E. G. Ström, and F. Brännström, "Cluster-based radio resource management for D2D-supported safety-critical V2X communications," IEEE Transactions on Wireless Communications, vol. 15, no. 4, pp. 2756-2769, Apr. 2016.

[8] A. Orsino et al., "Direct connection on the move: characterization of user mobility in cellular-assisted D2D systems," in IEEE Vehicular Technology Magazine, vol. 11, no. 3, pp. 38-48, Sept. 2016.

[9] K. Zheng, F. Liu, Q. Zheng, W. Xiang, and W. Wang, "A graphbased cooperative scheduling scheme for vehicular networks," IEEE Transactions on Vehicular Technology, vol. 62, no. 4, pp. 1450-1458, Feb. 2013.

[10] Q. Yang, B. Zhu, and S. Wu, "An architecture of cloud-assisted information dissemination in vehicular networks," IEEE Access, vol. 4, pp. 2764-2770, May 2016.

[11] T. Kim and M. Dong, "An iterative Hungarian method to joint relay selection and resource allocation for D2D communications," IEEE Wireless Communications Letters, vol. 3, no. 6, pp. 625-628, Dec. 2014.

[12] L. Wang and $\mathrm{H}$. Wu, "Fast pairing of device-to-device link underlay for spectrum sharing with cellular users," IEEE Commun. Lett., vol.18, no. 10 , pp.1803-1806, Oct. 2014

[13] B. Bai, W. Chen, K. Letaief, and Z. Cao, "A unified matching framework for multi-flow decode-and forward cooperative networks," IEEE JSAC, vol. 30, no. 2, pp. 397-406, Feb. 2012.

[14] H. Zhang, L. Song, and Z. Han, "Radio resource allocation for device-todeviec underlay communication using hypergraph theory," IEEE Transactions on Wireless Communications, vol. 15, pp. 4852-4861, July 2016.

[15] L. Wang, H. Wu, Y. Ding, W. Chen, and H. V. Poor, "Hypergraph based wireless distributed storage optimization for cellular D2D underlays," IEEE JSAC, vol. 34, no. 10, pp. 2650-2666, Oct. 2016.

[16] B. Bai, L. Wang, Z. Han, W. Chen, and T. Svensson, "Caching based socially-aware D2D communications in wireless content delivery networks: a hypergraph framework," IEEE Wireless Communication Magazine, vol. 23, no. 4, pp. 74-81, Aug. 2016.

[17] B. Azari, O. Simeone, U. Spagnolini, and A. M. Tulino, "Hypergraphbased analysis of clustered co-operative beamforming with application to edge caching," IEEE Wireless Communications Letters, vol. 5, no. 1, pp. 84-87, Feb. 2016.

[18] L. Militano, A. Orsino, G. Araniti, M. Nitti, L. Atzori, and A. Iera, "Trust-based and social-aware coalition formation game for multihop data uploading in 5G systems," Computer Networks, vol. 111, 24 Dec. 2016, pp. 141-151, ISSN 1389-1286, http://dx.doi.org/10.1016/j.comnet.2016.08.001

[19] T. Oosterwijk, "On local search and LP and SDP relaxations for k-set packing," arXiv preprint arXiv:1507.07459, 2015.

[20] C. A. J. Hurkens and A. Schrijver, "On the size of systems of sets every $\mathrm{t}$ of which have an SDR, with an application to the worst-case ratio of heuristics for packing problems," SIAM Journal of Discrete Mathematics, vol. 2, no. 1, pp. 68-72, 1989.

[21] B. Chandra, "Greedy local improvement and weighted set packing approximation," Journal of Algorithms, vol. 39, no. 2, pp. 223-240, May 2001.

[22] P. Berman, "A d/2 approximation for maximum weight independent set in d-claw free graphs," Algorithm Theory-SWAT 2000. Springer Berlin Heidelberg, Bergen, Norway, pp. 214-219, July 2000.

[23] S. R. Tiourine, C. A. J. Hurkens, and J. K. Lenstra, "Local search algorithms for the radio link frequency assignment problem," Telecommunication Systems, vol. 13, no. 2, pp. 293-314, 2000.

[24] A. Petcu and B. Faltings, "A value ordering heuristic for local search in distributed resource allocation," in Recent Advances in Constraints. Springer, pp. 86-97, 2005.

[25] M. T. Islam, A. M. Taha, S. Akl, and S. Choudhury, "A local search algorithm for resource allocation for underlaying device-to-devie communications," in Proc. 2015 IEEE Global Communications Conference, San Diego, CA, USA, Dec. 2015, pp. 1-6.

[26] V. I. Voloshin, "Introduction to Graph and Hypergraph Theory," Nova Science Publishers, Inc., 2009.

[27] E. Hazan, S. Safra, and O. Schwartz, "On the hardness of approximating k-dimensional matching," Electronic Colloquium on Computational Complexity, vol. 10, pp. 83-97, 2003.

[28] "Simulation Guidelines," ICT-317669-METIS/D6.1, METIS deliverable D6.1, Oct. 2013, [Online]. Avalible: http://www.metis2020.com/documents/deliverables/ 